\title{
A Feature Level Extraction based on Iris Recognition for Secure Biometric Authentication
}

\author{
Gourav Sachdeva \\ M.Tech, Information technology \\ Chandigarh engineering college Landran
}

\author{
Bikrampal Kaur, PhD \\ Professor, Information technology \\ Chandigarh engineering college Landran
}

\begin{abstract}
Iris recognition is most precise and consistent biometric identification system accessible in the current situation. The demand for an accurate biometric system that provides dependable identification and verification of an individual has increased over the years. A biometric system that provides reliable and accurate identification of an individual is an iris recognition system. This reliability is provided by unique patterns of human iris which differs from person to person up to an extent of identical twins having different iris patterns. This paper has proposed the hybridization of Hough Circular Transform, Scale Invariant Feature Transform and Genetic Algorithm. The genetic algorithm is applied to optimize the features set as obtained by Scale invariant feature transform.
\end{abstract}

\section{Keywords}

Iris Recognition, Hough Transformation, SIFT, Genetic Algorithm.

\section{INTRODUCTION}

Imagine how advantageous it would be to actuate the security alert at your home with the touch of a finger, or to enter your home by simply putting your hand on the entryway handle [1]. How might you want to stroll up to a close-by ATM which will check your iris so you can withdraw cash while never embeddings a card or entering a PIN? You will essentially have the capacity to obtain entrance to all that you are approved to, by exhibiting yourself as your personality. So, authentication of the biometrics is the way to solve above problems [2]. This situation may not be as distant as can be anticipate. Sooner rather than later, there might be no more utilize passwords and PIN numbers to confirm ourselves. These strategies have ended up being in secure and hazardous consistently. Innovation has acquainted a much more quick witted arrangement with us: Biometrics. Biometric confirmation will help in upgrading the security base against some of these dangers. Truth be told, physical qualities are not something that can be lost, overlooked or went starting with one individual then onto the next. They are greatly difficult to manufacture and a future criminal would reconsider before carrying out a wrong doing including biometrics. The idea of Iris Recognition was initially proposed by Dr. Forthright Burch in 1939.It was initially actualized in 1990 when Dr. John Daugman made the algorithms for it. These algorithms utilize methods of pattern recognition and some scientific calculation for iris acknowledgment. Iris acknowledgment is a system for biometric validation that uses methods of pattern recognition methods taking into account high-resolution pictures of the irises of a singular's eyes [3]. Iris is a muscle inside the eye that controls the extent of understudy, controlling the measure of light that controls the eye.

\subsection{Features of iris}

It is the colored portion (brown or blue) of the eye that regulates the size of the pupil. The coloration and structure of two irises is genetically linked but the details of patterns are not. They have stable and distinctive features for personal identification. They are stable with age. Extremely data rich physical structure having large number of features. Its inherent isolation and protection from the external environment. The outlandish possibility of surgically adjusting it without unsuitable danger to vision [4].

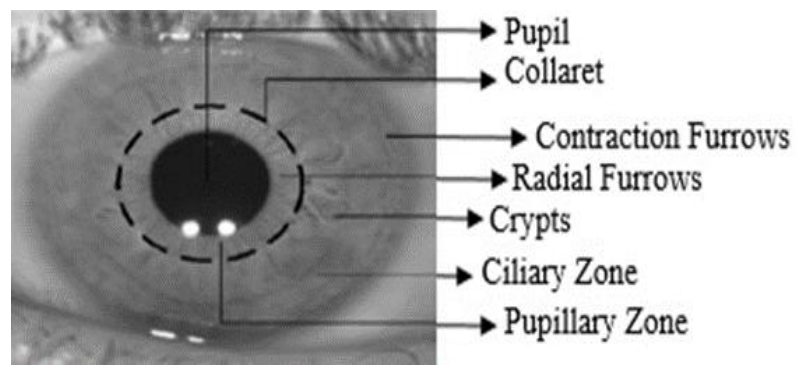

Figure 1 Front perspective of the eye

\subsection{Proposed Iris Recognition System}

This section will describe about the proposed model of iris recognition system:

Iris acknowledgment is viewed as a standout amongst the most solid and exact biometric acknowledgment framework accessible. A conventional IRIS acknowledgment methodology approximates iris limits as circles [5]. Change of the ring-formed district of the iris to a rectangular picture.

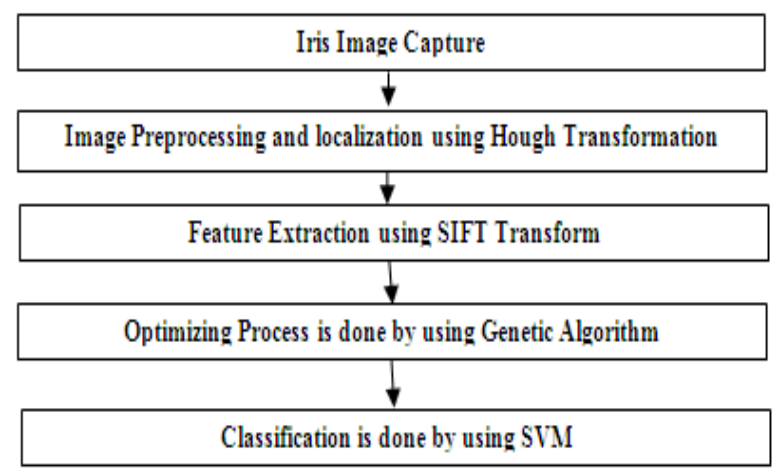

Figure 2: Stages in IRIS Recognition

Features are then removed from the rectangular standardized iris design (Gabor channels, log-Gabor channels, Gaussian channels, Laplacian-of-Gaussian channels, wavelet changes, and so on.). 


\subsubsection{IMAGE ACQUISITION}

It manages with capturing of a high quality image of the iris. The iris image ought to be rich in iris texture as the feature extraction stage relies on the picture quality. Therefore, the database obtaining has been done from online website.

\subsubsection{Image Localization Using Hough Circular Transform Algorithm}

Iris localization is a procedure to segregate the iris area from the rest of the acquired image. Iris can be approximated by two circles, one for iris/sclera boundary and another for iris/pupil limit. The Hough transform can be connected to identify the vicinity of a round shape in a given picture. It is utilized to distinguish any shape or to find the iris in the face [6]. The trademark mathematical statement of a circle of radius $r$ and center $\left(a_{1}, b_{1}\right)$ is given by:

$$
\left(x_{1}-a_{1}\right)^{2}+\left(y_{1}-b_{1}\right)^{2}=r^{2}
$$

This circle can be depicted by the two following mathematical statements:

$$
\begin{aligned}
& \mathrm{x}_{1}=\mathrm{a}_{1}+\mathrm{r} \cos (\mathrm{Q}) \\
& \mathrm{y}_{1}=\mathrm{b}_{1}+\mathrm{r} \sin (\mathrm{Q})
\end{aligned}
$$

Subsequently, the part of the Hough transform is to search for the triplet of parameters $\left(a_{1}, b_{1}, r\right)$ which decides the points. Two cases may be exhibited as depicted:

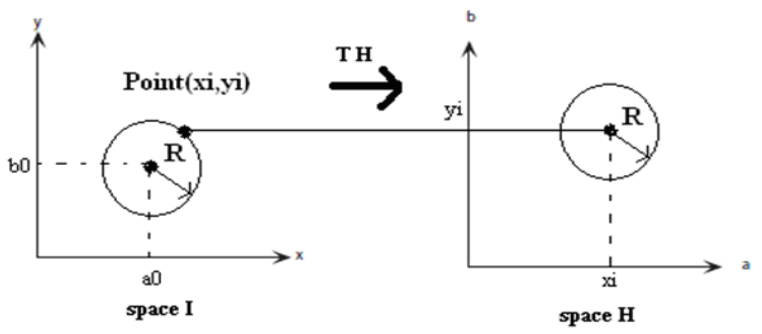

Figure 3: Hough Transformation

\section{A. Case of known radius}

On the off chance that one must know the radius of the circle to be identified in the picture, the parameter to search is decreased to a pair $\left(\mathrm{a}_{1}, \mathrm{~b}_{1}\right)$ and the $\mathrm{H}$ space is 2 dimensional. Consider a circle of radius $\mathrm{R}$ and center $\left(\mathrm{a}_{1}, \mathrm{~b}_{1}\right)$, the transformation for each point $\left(\mathrm{x}_{1}, \mathrm{y}_{1}\right)$ in space I yields a circle in space $\mathrm{H}$ having a center $\mathrm{x}_{1}, \mathrm{y}_{1}$ and radius $\mathrm{R}$.

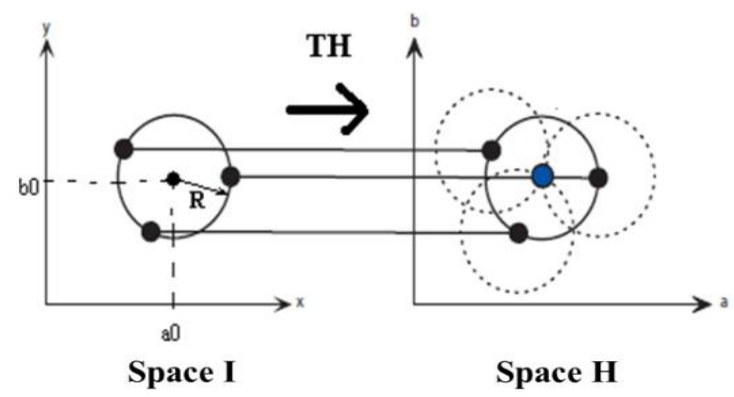

Figure 4: Detection Centroid of Circle Using Hough

Correspondingly, transformation of all points of the circle in image will be done. The outcome will be more circles where their intersection is the point $\left(a_{1}, b_{1}\right)$. This point is acquired via looking the maximum of the accumulator.
B. Case of unknown radius

For this situation, the work comprises to discover the triplet parameters $\left(a_{1}, b_{1}, r\right)$ characterize the purposes of circle to discover. The space will be in $3 \mathrm{D}$. For every point $\left(\mathrm{x}_{1}, \mathrm{y}_{1}\right)$ of the

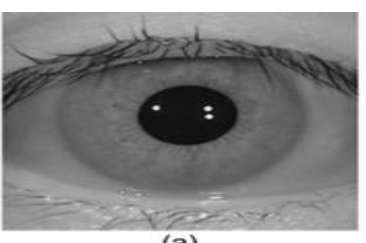

(a)

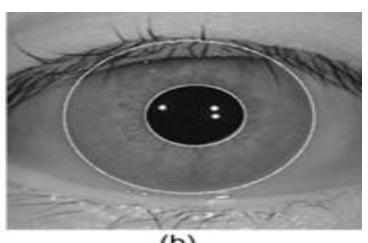

(b)
Figure 5: Detection of Inner and Outer Parts of IRIS

space I will coordinate a cone in space $\mathrm{H}$, as the radius $\mathrm{r}$ fluctuated from 0 to a given value (figure above). After transforming of all points of contour in the same way, the intersection will give a spherical surface corresponding to the maxim of accumulator. The region is portrayed by a center $\left(a_{1}, b_{1}\right)$ and the radius $r$ searched.

\subsubsection{Feature Extraction Using Sift Transform Algorithm}

Scale Invariant Feature Transformation (SIFT) [7] was initially created for universally useful object recognition. SIFT recognizes stable feature points of an item such that the same article can be perceived with invariance to illumination, scale, rotation and affine transformations. A brief portrayal of the ventures of the SIFT operator and their utilization in iris recognition is given next.

\section{a. Scale-Space Local Extreme Detection}

The first step is to build a Gaussian scale space, which is completed by convolving a variable scale 2D Gaussian operator $G\left(x_{1}, y_{1}, \sigma\right)$ with the input picture

$I\left(x_{1}, y_{1}\right): L\left(x_{1}, y_{1}, \sigma\right)=G\left(x_{1}, y_{1}, \sigma\right) * I\left(x_{1}, y_{1}\right)$

Difference of Gaussian images $D\left(x_{1}, y_{1}, \sigma\right)$ are then obtained by subtracting subsequent scales in each octave:

$D\left(x_{1}, y_{1}, \sigma\right)=L\left(x_{1}, y_{1}, k \sigma\right)-L\left(x_{1}, y_{1}, \sigma\right)$

Where $k$ is a constant multiplicative factor in scale space. Local extreme are then detected by observing each image point in $D(x ; y ; \sigma)$. A point is decided as a local minimum or maximum when its value is smaller or larger than all its surrounding neighboring points.

\section{b. Accurate Key point Localization}

Once a key point competitor has been found, in the event that it saw to have low contrast (and is along these lines delicate to noise) or on the off chance that it is localized along an edge, it is uprooted because it cannot be dependably recognized again with little variety of perspective or lighting changes. Two thresholds are utilized, one to prohibit low contrast points and other to exclude edge points [8].

\section{c. Orientation Assignment}

An introduction histogram is shaped from the gradient orientations inside a $16 \times 16$ locale around every key point. The introduction histogram has 36 bins covering the 360 degree range of orientations Every specimen added to the histogram is weighted by its gradient magnitude and by a Gaussian weighted circular window centered at the key point. The significant introductions of the histogram are then appointed to the key point, so the key point descriptor can be spoken to with respect to them, in this way accomplishing invariance to picture rotation. 


\section{d. Key point descriptor}

In this stage, a particular descriptor is registered at every key point. The picture gradient magnitudes and introductions, with respect to the significant introduction of the key point, are examined inside a 16X16 district around every key point. These specimens are then amassed into orientation histograms summarizing the contents over $4 \mathrm{X} 4$ sub regions. Every introduction histogram has 8 bins covering the 360 degree range of orientations. Each sample added to the histogram is weighted by its gradient magnitude and by a Gaussian circular window centered at the local extremism. The descriptor is then formed from a vector containing the values of all the orientation histogram entries therefore having a $4 \mathrm{X} 4 \mathrm{X} 8=128$ element feature vector for each key point.

\section{e. Key point matching}

Matching between two images $I_{1}$ and $I_{2}$ is performed by comparing each local extreme based on the associated descriptors. Given a feature point $p_{11}$ in $I_{1}$, its closest point $p_{21}$, second closest point $p_{22}$, and their Euclidean distances $d_{1}$ and $d_{2}$ are calculated from feature points in $I_{2}$. If the ratio $d_{1} / d_{2}$ is sufficiently small, then point's $p_{11}$ and $p_{21}$ are considered to match. Then, the matching score between two images can be decided based on the number of matched points. According to [7], in this work the chosen value of threshold is 0.76 for the ratio $d_{1} / d_{2}$

\section{f. Trimming of false matches}

The key point matching procedure described may generate some erroneous matching points. The spurious matching points can be removed using geometric constraints [9]. Then limit typical geometric variations to small rotations and displacements. Therefore, when placed two iris images side by side and draw matching lines true matches must appear as parallel lines with similar lengths. According to this observation, compute the predominant orientation $Q_{P}$ and length $l_{p}$ of the matching, and keep the matching pairs whose orientation $\mu$ and length ' are within predefined tolerances $\epsilon_{Q}$ and $\epsilon_{P}$ so that $\left|Q-Q_{P}\right|<\epsilon_{Q}$ and $\left|l-l_{P}\right|<\epsilon_{l}$.

\subsubsection{Genetic Algorithm}

As a preparation to start the optimization process, a Genetic Algorithm, requires a group of initial solutions as the first generation. The first generation is usually a group of randomly produced solutions created by a random number generator [6]. The population, which is the number of individuals in a generation, should be big enough so that there could be a reasonable amount of genetic diversity in the population. Also, it should be small enough for each generation to be computed in a reasonable period of time using the computer resources available. Typically, a population includes individuals between 20 and 100. The fitness function is evaluated to measure how close that the individuals fit the desired result. A fitness function could be either complex or simple depending on the optimization problem addressed. In a case of minimization problem, the most fitted individuals will have the lowest numerical value of the associated fitness function.

In GAs, there are mainly two ways to select a new population: Roulette Wheel Selection (RWS) and Stochastic Universal Sampling (SUS). The individuals will be recombined (crossover) after the selection. This operation is to produce two new individuals from two existing individuals selected by the operator of selection by cutting them at one or more position and exchanging the parts following the cut. The new individuals therefore can inherit some parts of both parents' genetic material. There are usually four ways of doing this: one point crossover, two-point crossover, cycle crossover and uniform crossover [10]. The difference is that the new individual is produced from a single old one. In this operation, the bit values of each individual are randomly reversed according to a specified property. A mutation can also help the GA to avoid local optimums. The features are reduced to 100-115 after applying genetic algorithm. Now the samples are saved in the database. This is the testing part of the recognition system.

\subsection{Save in $d b$}

In the last step saving of the templates will be done in database.

\section{FLOWCHART}

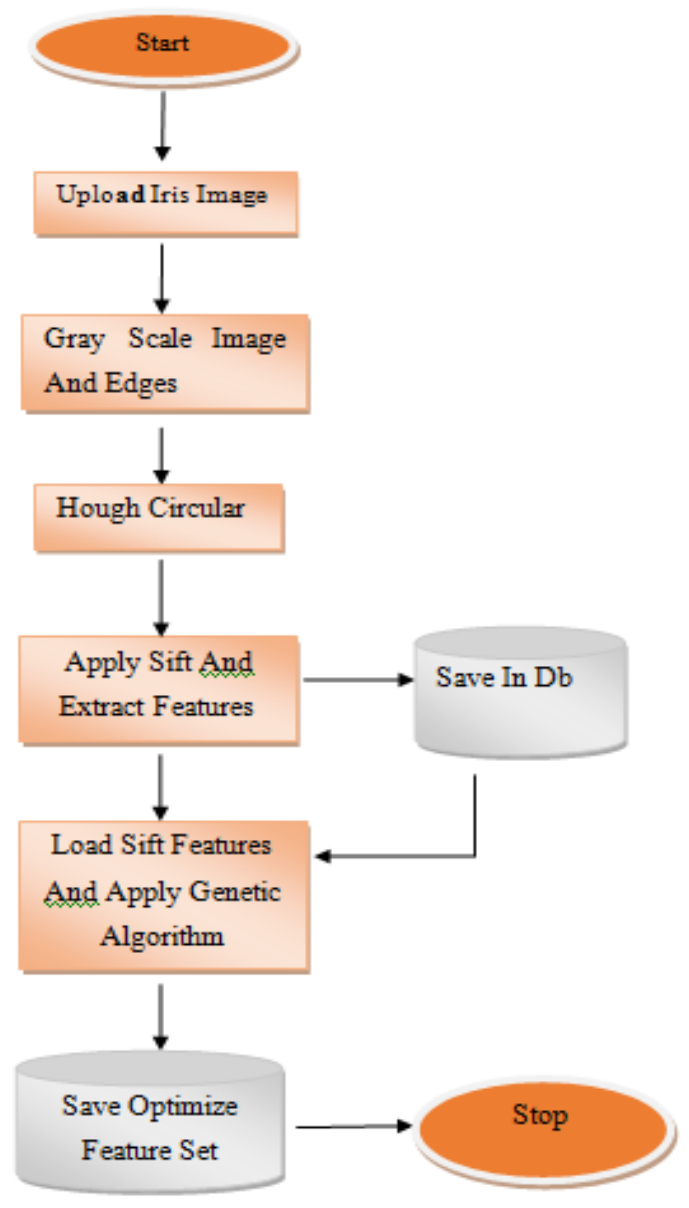

Figure 6: Methodology of Iris Recognition 


\section{RESULT ANALYSIS}

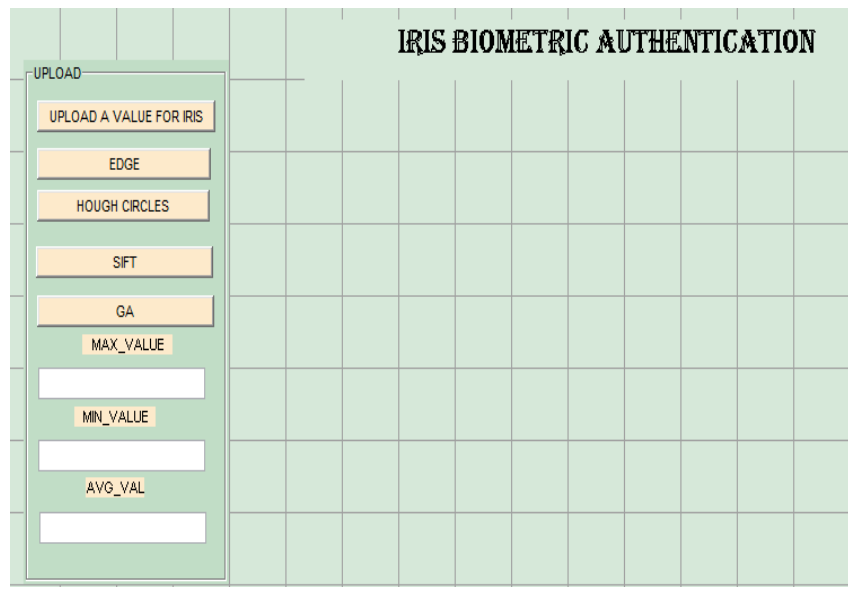

Figure 7: Proposed User Interface

The above figure shows the graphical user interface of the proposed system with different user interface controls like pushbuttons, edit text and static text.

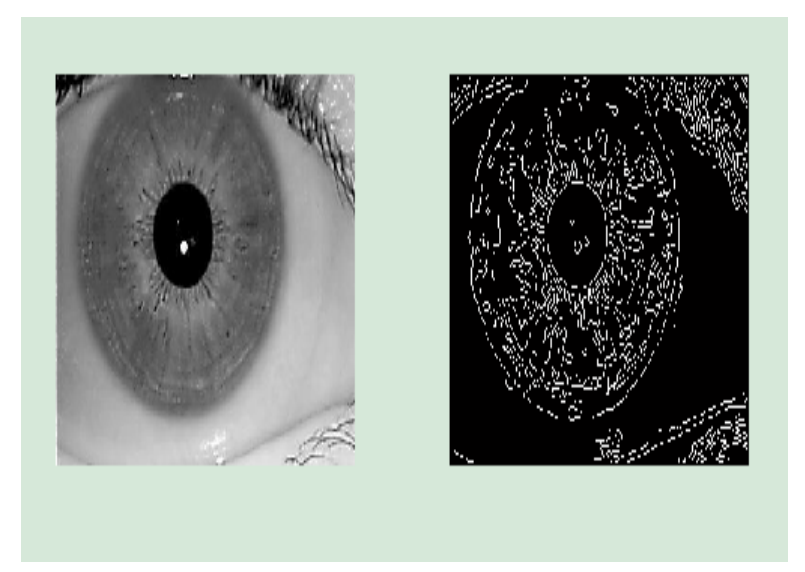

Figure 8: Iris uploaded Image

The above figure 8 shows the uploaded image of iris with edge detection which is used to find the number of edges using canny edge detector. The canny edge detector is one of the major edge detection techniques. There are also some other techniques to find the edges like sobel, prewitt, log etc.

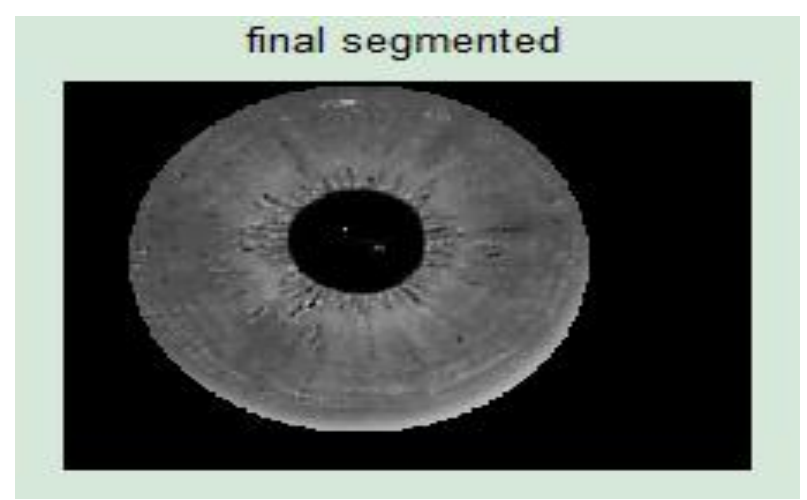

Figure 9: segmented image
The above figure shows the final segmented image of iris after applying Hough circular transform to find the inner and outer radius of the pupil.

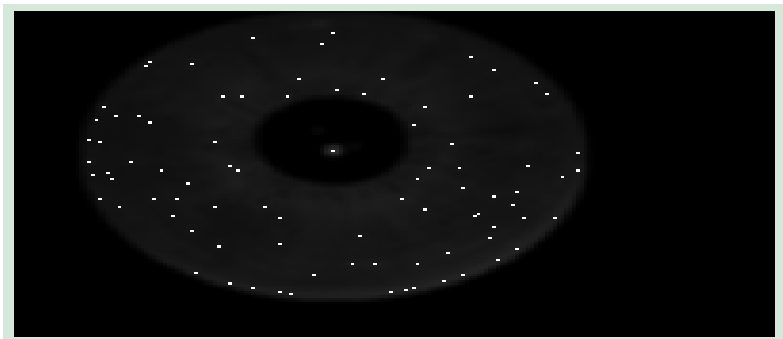

\section{Figure 10: SIFT image}

The above figure shows the key points on the uploaded image after applying scale invariant feature transform. These are the key features that are used for the feature vector that are saved in the database of MATLAB. That database file is having .mat extension

\begin{tabular}{|c|c|}
\hline 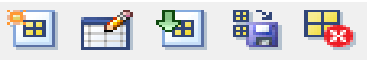 & Stack: $\backsim$ plot(data. \\
\hline Name - & Value \\
\hline 日) data_new & $<1 \times 500$ double $>$ \\
\hline
\end{tabular}

Figure 11: SIFT database

The above figure shows the feature extraction database of the uploaded image which is having 1 row and 500 column values that contains the feature values

\begin{tabular}{|c|c|}
\hline 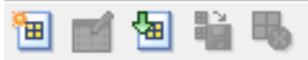 & Q9 Select data to plot \\
\hline Name - & Value \\
\hline$\boxplus$ Gareduced & $<1 \times 50$ double $>$ \\
\hline
\end{tabular}

Figure 12: Reduced feature set

The above figure shows the Genetic reduced feature set after applying Scale Invariant feature transform which is reduced to 50 values from 500 values. The above figure shows the matrix of 1 row and 50 columns of the uploaded iris image and shows the optimize feature set.

\section{CONCLUSION}

In the proposed system a new technique is generated for iris verification system to increase the accuracy of the authentication systems using hybridization of various methods. In this work utilization of Hough Circular Transform algorithm for image localization, SIFT Transform algorithm for feature extraction, genetic algorithm for optimizing the extracted feature has been done. It has been also concluded that proposed work leads to the enhancement of the accuracy of the work.

Future works could go in the direction of using more robust modelling techniques by doing hybridization of Support vector machine with another various techniques. 


\section{REFERENCES}

[1] L. Ma, T. Tan, Y. Wang, and D. Zhang, "Personal identification based on iris texture analysis," IEEE Trans. Pattern Anal. Mach. Intell., vol. 25,no.12, pp.15191533,Dec.2003.

[2] Z. Sun, T. Tan, and Y. Wang, "Robust encoding of local ordinal measures: A general framework of iris recognition," in Proc. ECCV Workshop Bio AW, 2004, pp.270-282.

[3] C. Sanchez-Avila and R. Sanchez-Reillo, "Two different approaches for iris recognition using Gabor filters and multiscalezerocrossingrepresentation,"PatternRecognit.,v ol.38,no.2,pp.231240,Feb.2005

[4] K. Miyazawa, K. Ito, T. Aoki, K. Kobayashi, and H. Nakajima, "An effective approach for iris recognition using phase-based image matching," IEEE Trans. Pattern Anal. Mach. Intell., vol. 30, no. 10, pp. 17411756,Oct.2008

[5] L. Birgale and M. Kokare, "Iris recognition without iris normalization,"J.Comput.Sci.,vol.6,no.9,pp.10421047,2010
[6] C. Belcher and Y. Du, "Region-based SIFT approach to iris recognition,”Opt.LasersEng.,vol.47,no.1,pp.139147,Jan.2009.

[7] [7].S. Shah and A. Ross, "Iris segmentation using geodesic active contours," IEEE Trans. Inf. Forensics Security, vol. 4, no. 4, pp. 824-836, Dec. 2009.

[8] K. Roy, P. Bhattacharya, and C. Y. Suen, "Towards non ideal iris recognition based on level set method, genetic algorithms and adaptive asymmetrical SVMs," Eng. Appl. Artif. Intell., vol. 24, no. 3, pp. 458-475,Apr. 2011.

[9] R. Zhu, J. Yang, and R. Wu, "Iris recognition based on local feature pointmatching," in Proc. Int. Symp. Commun. Inf. Technol., 2006, pp. 451-454.

[10] Kulkarni S. B., Shirodkar S., Hegadi R. S., Kulkarni U. P., Iris Recognition using Fusion of Gray Level Cooccurrence Matrix and Gray Level Run Length Matrix, ICSIP 2013, Elsevier, pp. 241 - 246, 2013. 